\title{
Genotyping the Baboon ABO Histo-Blood Group Locus by Two-Color Fluorescence SSCP
}

BioTechniques 27:1054-1062 (November 1999)

\author{
David C. Diamond, Katalin \\ Illes, Leonard L. Bailey and \\ Aladar A. Szalay \\ Loma Linda University and \\ Medical Center, Loma Linda, \\ CA, USA
}

\begin{abstract}
The use of baboons in experimental medicine, especially as organ and tissue donors, would be facilitated by the availability of $A B O$ histo-blood group $O$ animals, which are currently rare. To meet our need in breeding and identifying such animals, we have developed a single-stranded conformational polymorphism (SSCP)based genotyping assay using fluorescence detection. Various buffers and labeling schemes were evaluated to optimize allele discrimination. Through the use of a nested PCR protocol, single-cell sensitivity was achieved, making the assay applicable to preimplantation diagnosis following in vitro fertilization. We discuss the comparative advantages of SSCP vs. alternative methodologies for genotyping.
\end{abstract}

\section{INTRODUCTION}

We are interested in breeding $\mathrm{ABO}$ histo-blood group $\mathrm{O}$ baboons because of their potential usefulness as surrogates for humans in medical research or as donors in xenotransplantation. Group $\mathrm{O}$ baboons are rare in nature, but we have recently been able to define $\mathrm{O}$ and other alleles of the baboon $\mathrm{ABO}$ locus by comparative sequence analysis (5). To identify $\mathrm{O}$ homozygous embryos from in vitro fertilization (IVF) for implantation, and $\mathrm{O}$ heterozygotes for natural breeding, we have developed a twocolor fluorescence polymerase chain reaction (PCR) single-stranded conformational polymorphism (SSCP) genotyping assay. We compared internal and external labeling, a variety of buffer conditions and used nested PCR to achieve sufficient sensitivity to analyze single cells. For electrophoresis and fluorescence detection, we used a Model 373A/XL DNA Sequencer with data analysis by GENESCAN ${ }^{\circledR} 2.1$ software (PE Biosystems, Foster City, CA, USA). Although SSCP has enjoyed widespread use in scanning for mutants generally $(2,16,17)$, it has found much less application in the discrimination of known sequences (i.e., genotyping). We found that SSCP offered several advantages over allele-specific PCRand oligonucleotide hybridizationbased methodologies, particularly in terms of ease of development and tolerance of previously unobserved alleles.

$\mathrm{ABO}$ histo-blood group phenotype is controlled by a single locus encoding allelic glycotransferases (24). O alleles fail to express an active enzyme and so are recessive. Thus, in $\mathrm{AO}$ and $\mathrm{BO}$ heterozygotes, $\mathrm{O}$ alleles can only be detected by genotyping. Because $\mathrm{O}$ homozygotes express neither an A nor B antigen, they constitute a universal donor type, which facilitates their use as blood or organ donors either in experimental or therapeutic settings.

The activity expressed by alleles of the baboon $\mathrm{ABO}$ locus can generally be predicted by examining a <300-nucleotide (nt) segment of the major (7th) exon of the gene (5). Nucleotide dimorphisms at N796 and N803 (numbering according to References 3 and 23) control substrate binding by the enzymes in all examined primates $(5,6,10,14)$, thereby conferring A or B transferase activity, and in baboon DNA, are associated with an additional dimorphism at N813. The major $\mathrm{O}$ allele of baboon DNA, which has an A-like sequence at the above three positions, has four nucleotide differences from the prototype A and B alleles at positions N629, N651, N704 and N711 (5). However, the O-like sequences at the latter two positions are occasionally observed in functional alleles. In contrast, the major $\mathrm{O}$ allele in human DNA is the result of a nucleotide deletion located in the 6th exon $(1,25)$, precluding the use of a single amplified DNA segment in SSCP as it would be too large. Here, we describe the finding that these various alleles can be readily identified by SSCP, thereby providing a rapid and sensitive assay for the $\mathrm{O}$ allele and for genotyping generally.

\section{MATERIALS AND METHODS}

\section{Primers}

A pair of PCR primers, GTRF (5'CGAGGTGGATTACCTGGTGT-3') and GTRR (5'-ATCATGGCCTGGTGGCAG-3'), positioned shortly upstream and downstream, respectively, of the dimorphic nucleotide positions noted above were designed using Primer3 on the MIT Web site (http://www. genome.wi.mit.edu/cgi-bin/primer/ 
primer3. cgi) and further evaluated using Amplify 1.2 (Bill Engels, University of Wisconsin, Madison, WI, USA). The $5^{\prime}$ ends of these primers are at N606 and N866, giving rise to a $261 \mathrm{bp}$ PCR product. The primers UPF $\left(5^{\prime}-\right.$ TGGGCCACCGTGTCCACTACTATGTCTT-3') and LPC (5'-TCCGGACCGCCTGGTGGTTCTTG-3') used in the first stage of nested PCR have been described previously (5); their $5^{\prime}$ ends are at N425 and N1057, respectively, demarking a 633 bp product. Primers were synthesized by the CMBGT Core Facility (Loma Linda, CA, USA), and labeled primers were purchased from GENSET (La Jolla, CA, USA).

\section{DNA Isolation and Amplification}

DNA was isolated from 2-10 $\times 10^{6}$ peripheral blood lymphocytes using the QIAamp ${ }^{\circledR}$ Blood Kit (Qiagen, Valencia, CA, USA). Approximately $200 \mathrm{ng}$ of DNA were used as template in a $50 \mu \mathrm{L}$ PCR amplification containing $1.5 \mathrm{mM}$ $\mathrm{MgCl}_{2}, 400 \mathrm{nM}$ and $900 \mathrm{nM}$ primers GTRF and GTRR, respectively, $200 \mu \mathrm{M}$ each of the 4 dNTPs, $1.5 \mathrm{U}$ of AmpliTaq Gold $^{\mathrm{TM}}$ (PE Biosystems) and the supplied buffer. For internal labeling, 0.25 $\mu \mathrm{L}$ of $100 \mu \mathrm{M}$ R110-dUTP (PE Biosystems) was also added; otherwise, GTRF and GTRR labeled at their $5^{\prime}$ ends with HEX or FAM, respectively, were used.

Amplification was carried out on a GeneAmp ${ }^{\circledR}$ PCR System 2400 (PE Biosystems) using a touchdown PCR cycle: $94^{\circ} \mathrm{C}$ for $9 \mathrm{~min} ; 14$ cycles of $94^{\circ} \mathrm{C}$ for $10 \mathrm{~s}, 62^{\circ} \mathrm{C}$ for $30 \mathrm{~s}$ reduced $1^{\circ} \mathrm{C}$ each cycle at $72^{\circ} \mathrm{C}$ for $20 \mathrm{~s}$; and 25 cycles of $94^{\circ} \mathrm{C}$ for $10 \mathrm{~s}, 48^{\circ} \mathrm{C}$ for $30 \mathrm{~s}, 72^{\circ} \mathrm{C}$ for $20 \mathrm{~s}$. The final extension step was extended by $7 \mathrm{~min}$ to assure complete elongation. The initial denaturation was necessary to activate the polymerase. Success of the reaction was monitored by agarose gel electrophoresis, and the reaction product was purified using a QIAquick $^{\mathrm{TM}}$ Kit (Qiagen) before SSCP. Templates giving poor amplification were subjected to nested PCR.

\section{PCR from Single Cells}

Various serial dilutions of cultured cells of the baboon lymphoblastoid cell line 26-CB-1 (ATCC, Rockville, MD, USA) were either used directly or plat- ed in a microdish. In the latter case, droplets observed by microscopy to contain the designated number of cells could be then transferred, in $2 \mu \mathrm{L}$, to PCR tubes and lysed by addition of 1 $\mu \mathrm{L}$ of $17 \mu \mathrm{M}$ sodium dodecyl sulfate (SDS), $2 \mu \mathrm{L}$ proteinase $\mathrm{K}(125 \mu \mathrm{g} / \mathrm{mL})$ and incubation at $37^{\circ} \mathrm{C}$ for $60 \mathrm{~min}$. Following incubation at $95^{\circ} \mathrm{C}$ for $15 \mathrm{~min}$ to inactivate the proteinase $\mathrm{K}$, PCR buffer, $\mathrm{MgCl}_{2}$, dNTPs, enzyme and the primers UPF and LPC were added, and amplification was carried out essentially as described above except for using 35 cycles at the final extension, and the primers were equimolar at $400 \mu \mathrm{M}$. One microliter of this reaction was then used as template in the GTRF-GTRR reaction described above.

\section{SSCP}

The fluorescent-dye-labeled amplification product $(0.3-2 \mu \mathrm{L})$ was combined with $4.2 \mu \mathrm{L}$ of $100 \%$ deionized formamide: $500 \mathrm{mM}$ EDTA (20:1) and $0.8 \mu \mathrm{L}$ of GENESCAN-350 ROX size markers. The samples were then denatured by heating at $95^{\circ} \mathrm{C}$ for $2 \mathrm{~min}$ and immediately cooling them in ice water. Once cold, the samples were loaded onto a $0.4 \times \mathrm{MDE}^{\mathrm{TM}}$ Acrylamide Gel (FMC Bioproducts, Rockland, ME, USA) of standard length ( $24 \mathrm{~cm}$ wellto-read) with a 24-well shark's-tooth comb on a Model 373A or 373XL DNA Sequencer (both from PE Biosystems). The gel buffer was one of the following: TBE (90 mM Tris-borate, $\mathrm{pH} \mathrm{8.3,4}$ mM EDTA), TBE supplemented with $5 \%$ glycerol or TME [ $60 \mathrm{mM}$ Tris- $\mathrm{HCl}$, $70 \mathrm{mM}$ 2,N-morpholinoethane sulfonic acid (MES), pH 6.8, 1 mM EDTA; or 35 mM Tris-HCl, 70 mM MES, pH 6.3, 1 mM EDTA]. Electrophoresis was performed in either TBE or TME, as appropriate, at $20 \mathrm{~W}$ for $12-13 \mathrm{~h}$. Gel file data were processed using GENESCAN Analysis 2.1.

\section{RESULTS}

\section{Effects of Different Buffer Components}

In our initial evaluation of SSCP, we used an internally labeled PCR product on the TBE gels. The A and B alleles were difficult to distinguish from each other; the $\mathrm{O}$ allele, however, was clearly separated from A and B, but did not undergo strand separation (data not shown). Because our primary interest was screening for $\mathrm{O}$ heterozygotes, we viewed this finding as an encouraging result. It has now become a common practice to include glycerol in SSCP buffers, as was done earlier for DNA strand separation (21); in fact, PE Biosystems recommends the inclusion of glycerol when preparing MDE gels for use on their systems. Including glycerol, we observed two distinct bands for each of the three primary alleles (Figure 1, lanes 11-13). The upper band was generally sharper and showed much better resolution, one allele to the next, than the lower band. The $\mathrm{O}$ allele upper band was still easily distinguished. However, an uncommon B allele, $\mathrm{Bv}$, which shares the O-like residues at positions N704 and N711, was difficult to characterize, particularly in the $\mathrm{BvO}$ heterozygote that was available. As became clear in two-color experiments, this difficulty arose from the (near) co-migration of bands.

It has been reported that more acidic MES-containing buffers improve discrimination between alleles, particularly for longer fragments (11). We tested MES-containing buffers at $\mathrm{pH} 6.8$ and $\mathrm{pH} 6.3$, and while we did see changes in the pattern of bands, overall differentiation of the particular fragments in this study was not improved (data not shown). Moreover, in our hands the buffer proved unstable, taking on a yellowbrown color over a period of 2-3 weeks, making it inconvenient to work with.

\section{Internal Labeling vs. Labeled Primers}

Because of its ease of use and flexibility in choice of primers, we initially used a dye-labeled dNTP. This proved serviceable in demonstrating the general usefulness of the technique, while avoiding the expense of labeled primers and has been applied successfully (9). However, the use of labeled primers did prove advantageous. Bands tend to be less broad because the single, defined position of the label results in a more homogeneous product. The two-color capability afforded by labeled primers immediately revealed that the upper 
bands (still blue) were the antisense strand, while the lower bands (now green) were the sense strand (Figure 1, lanes 2-9). Of greater consequence, the capability also facilitated characterization of the $\mathrm{Bv}$ allele, which had been difficult to characterize, as noted above. Using internal labeling with heterozygous samples, we clearly observed only the bands from the other allele. Even with a Bv homozygote, there appeared to be only a single broad band near the usual lower position (Figure 1, lane 14). With two colors, it became obvious that the broad lower band actually consisted of the antisense strand of the $\mathrm{Bv}$ allele running just between the $B$ and $B v$ sense strands (Figure 1, lane 9 and Figure 2, A and $\mathrm{B}$, lane 6).

Figure 2 shows SSCP analysis of a cohort of baboons that had not been previously genotyped. The gel image (Figure 2A) allows one to quickly see and evaluate a large amount of data. Chromatogram profiles (Figure 2B) of individual lanes allow for more careful consideration and have been relied on for conclusive determination of genotype. There is a striking contrast in the sharpness of the sense and antisense bands. Bandwidth can be influenced by the stability of single-strand conformation; multiple conformations in equilibrium result in broader bands than single stable conformations. However, since the antisense band of $\mathrm{Bv}$ (Figure 2B, lane 6) also shows some broadening, the speed of migration may also contribute to this effect. The chromatograms allow easier visualization of the broad, faint bands that the sense strand (green) often produces.

Compare, for example, the chromatograms for Figure 2B, lanes 3 and 6; the sense strand for $\mathrm{Bv}$ becomes manifest as a very broad band (green) at higher apparent molecular weight than the antisense (blue) band of $\mathrm{Bv}$. Because the scaling is set for the profile of each lane individually (note the different vertical axes in Figure 2B), lane-tolane comparison, even with relative over- or underloading, is facilitated. The smearing seen in Figure 2A, lanes 7 and 9 is likely due to some combination of overloading, mispriming in PCR, sample degradation and incomplete denaturation. Nonetheless, the positions of the primary bands in these lanes correctly identify the genotype, as confirmed by sequencing (data not shown).

\section{New Alleles Detected}

In addition to providing a rapid screen for the known alleles, previously unobserved alleles become readily apparent. For example, analogous to Bv, which has two O-like nucleotides (at N704 and N711), we have now observed an A-related allele, Av, that after sequencing shares the same two nucleotides. This allele also emphasizes the utility and importance of having two bands to examine. The blue (antisense) band for $\mathrm{O}$ and $\mathrm{Av}$ run at virtually the same position and could readily lead to misidentification. The green (sense) band for Av runs quite noticeably faster than any other (Figure 2, A and B, lane 13). Figure 2, lane 12 shows a blue band running midway between the positions of the $\mathrm{O}$ (also seen in lane 12) and $\mathrm{Bv}$ antisense (blue) strands. The amplified region of this allele differs from the $\mathrm{O}$ allele only in that $\mathrm{N} 813$ is a $\mathrm{G}$ instead of an $\mathrm{A}$, a silent mutation. This initially presented a conundrum since the amino acid sequence encoded by both alleles in this region was that of the $\mathrm{O}$ allele, yet the animal had an $\mathrm{A}$ phenotype; all animals used in this study had been previously phenotyped using immunofluorescence staining of buccal mucosa (15). Further sequencing revealed a $\mathrm{T}>\mathrm{C}$ change in one of the alleles at N932, encoding a Leu>Pro change, which we hypothesized, is a compensatory mutation restoring activity to that allele (AOR). Finally, we have observed an $\mathrm{O}$ allele with $\mathrm{G}$ instead of $A$ at N681 (Ov; Figure 2A, lane 9) and a B allele with $\mathrm{T}$ instead of $\mathrm{C}$ at N651 (Bv2; data not shown).

\section{Sensitivity for Single-Cell Genotyping}

Eventually we will apply this technique to one or a few cells dissected from in vitro fertilized embryos to choose which ones to implant $(7,20)$. Therefore, we tested the feasibility of using nested PCR to generate sufficient material for SSCP from a limited amount of template. We found that simply using available primers that amplify a major portion of the 7th exon as a first stage, followed by a second amplification with the primers chosen for SSCP, readily produced ample product using serially diluted genomic DNA (data not shown). Next, we tried the same amplification protocol with serially diluted 26-CB-1 cells. We found that lysis by SDS followed by proteinase K digestion (7), rather than simply heating in distilled water, was required to release adequate amounts of the template. Figure 3 shows successful amplification from even single cells. The presence of

Figure 1. Comparison of internal and end labeling protocols. The portion of a gel file showing the strand-separated PCR products from templates previously genotyped by sequencing; size markers present but not shown. Lanes 2-9, end label; lane 10, empty; lanes 11-14, internal label. Sample genotypes: lanes 2 and 12, BB; lane 3, AB; lanes 4, 5 and 11, AA; lane 6, AO; lanes 7 and 13, OO; lane 8, BO; lane 9, $\mathrm{BvO}$; lane 14, BvBv. Band positions for the A, B and $\mathrm{O}$ alleles are marked to the left and right sides of the gel image for end and internally labeled samples, respectively.

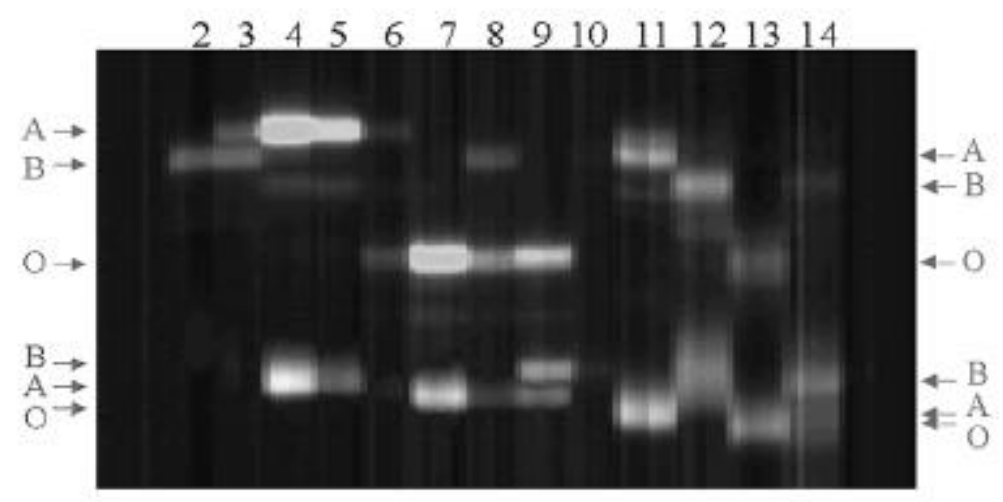



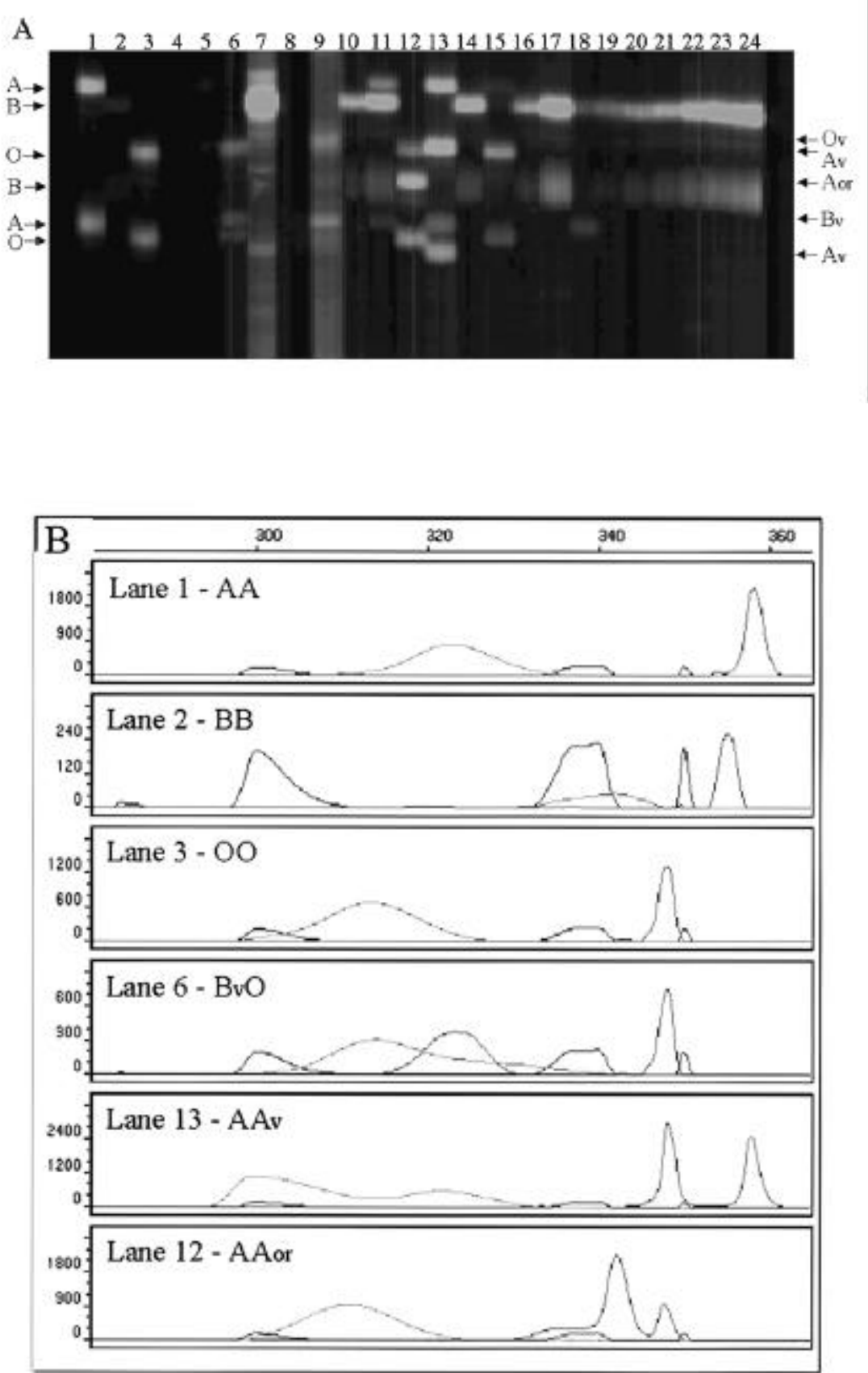

Figure 2. SSCP with samples of unknown genotype. (A) The portion of a gel image showing the strand-separated PCR products; size markers present but not shown. Lanes 1-6 (4 empty) are known standards: $\mathrm{AA}, \mathrm{BB}, \mathrm{OO}, \mathrm{AAv}$ and $\mathrm{BvO}$, respectively. Unknowns were interpreted as follows: $\mathrm{BB}$, lanes 7,10, 14, 16, 17, 19-24; lane 8, empty; lane 9, OvBv; lane 12, OAOR; lane 13, AAv; lane 15, AO; lane 18, $\mathrm{BBv}$. Lane 11 looks like $\mathrm{AB}$ but the phenotype is $\mathrm{A}$, so the second allele must be the rare, B-derived $\mathrm{O}$ allele described previously (1). Some bands are too faint to see at this exposure, but can be readily seen in chromatogram profiles (e.g., lane 2 in panel B). Band position for the A, B and $\mathrm{O}$ alleles are marked to the left, and some less common alleles are marked to the right sides of the gel image, respectively. (B) Chromatogram profiles of the same gel for lanes 1-3, 6, 13 and 12 (top to bottom). Note independent scaling of the vertical axis for each lane, allowing faint bands (e.g., in lane 2 ) to be readily discerned. Size markers in each lane, seen in red, were used to establish the common horizontal scale seen at the top of the figure. The process of aligning the chromatogram lanes according to the size standards has had the additional effect of compressing those bands with an apparent size $>340 \mathrm{nt}$ and spreading those bands with a smaller apparent size as compared to their appearance in the gel image. 
the expected approximate number of cells in each aliquot was confirmed microscopically. In other experiments, droplets containing the exact number of cells were used as described above. Generally, little difference in yield is seen over the range of initial template concentration. The one single-cell reaction with poor yield could be the result of a poor release of template, but it is not a failure and could be subjected to another round of amplification if it were a crucial sample.

\section{DISCUSSION}

Many genotyping methods have relied on specific detection of a defined set of alleles $(4,8,12,18,19,22)$. Thus, one needed a restriction site, probe, primer or similar entity (or at least a unique combination of them) for every allele. While unknown alleles might be signaled by an unexpected reactivity with such reagents, variation at sites in addition to those already defined would be, at best, undetected and could easily give misleading results. Moreover, arriving at a set of parameters suitable for all of the oligonucleotides that might be involved can be difficult. In SSCP, a set of oligonucleotide primers hybridizing to non-variant regions are used; thus, they interact the same with all alleles and can potentially detect all variants within the tested region. Also, the size of the set is not directly dependent on the number of alleles, although in cases where allelic variations are distant from each other, multiple primer sets may be needed for a complete assay. As with any method, SSCP will obviously give misleading results for any genotypes that differ only outside the tested region. In our system, this is exemplified by the rare, B-like $\mathrm{O}$ allele seen in Figure 2, lane 11. The allele is as yet incompletely characterized and can only be recognized by discordance between phenotype and apparent genotype.

The use of real-time, multicolor fluorescence detection on the Model 373A/XL DNA sequencer provides both advantages and drawbacks. The lack of any post-electrophoresis processing of the gel saves significant time and labor. The ability to clearly distinguish the two strands proved crucial for our analysis, ensuring that, in heterozygotes, co-migration of sense and antisense strands from different alleles could be detected. Because of the realtime detection, the width of bands is related to the speed of migration, and thus, electrophoresis conditions (i.e., acrylamide concentration, ionic strength and current) must be confined to a narrower range than other systems. The multicolor fluorescence gel scanners now on the market may allow a broader range of conditions to be used but at the cost of post-electrophoresis handling of the gel. This particular configuration of the 373A/XL DNA sequencer lacks thermostating, imposing a further constraint on the wattage that could be tolerated and allowing ambient conditions in the laboratory (which varied by $>10^{\circ} \mathrm{C}$ over the course of this study) to influence the run. We attribute much of the run-to-run variability we observed to the effect of ambient temperature on polymerization and electrophoresis. The inherent redundancy provided by the independent assessment of both single strands contributes to the robustness of allele discrimination. Serendipitously, in this particular system when resolution has diminished for one color, it has tended to increase for the other.

Kukita et al. (11) and Liu and Sommer (13) have tested the effect of alternate buffer components and reported superior results with MES and HEPES.
The consequence of using these components is to lower $\mathrm{pH}$, as is the case with glycerol. We did find that, compared with TBE, the lower $\mathrm{pH}$ buffers did improve overall resolution of the different alleles. Although the use of MES instead of glycerol clearly altered the relative mobilities of various alleles, no global advantage was noted for either our standard fragments or longer products (data not shown). It may be important that these previous studies $(11,13)$ made use of standard acrylamide rather than MDE acrylamide gel. Because they used autoradiography, their readout relied on distance migrated at one point in time, rather than the time required to migrate a set distance, as in our protocol. It seems clear to us that lower $\mathrm{pH}$ (compared to TBE) improves the discriminatory power of SSCP, but the optimum condition will depend on the sequences being analyzed, and no particular set of buffer components will assure universal optimization.

The ability of SSCP to rapidly identify previously uncharacterized alleles deserves emphasis. It is possible for SSCP to fail to detect a variant allele if it does not produce a resolvable change in mobility. However, the number of new alleles detected in this study leads us to believe that this has not been a significant problem in our system. In more than 40 animals previously examined by sequencing (Reference 5 and

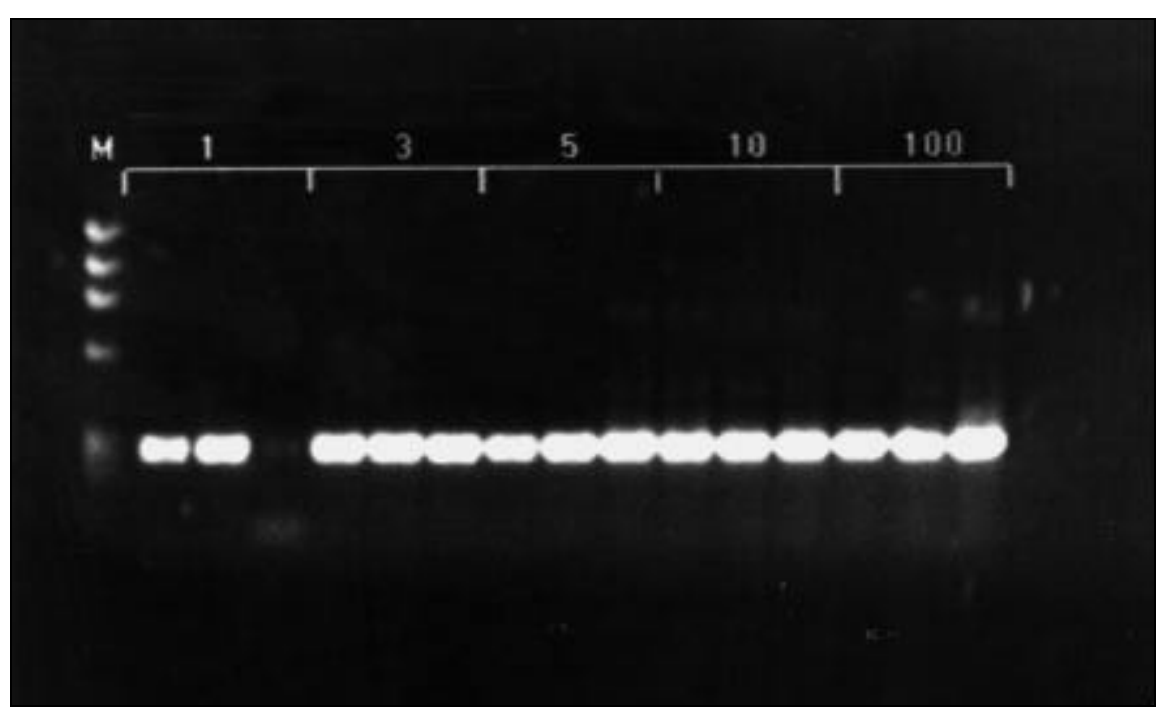

Figure 3. Template titration with nested PCR. Triplicate aliquots from serial dilutions of 26-CB-1 cells were subjected to nested PCR and agarose gel electrophoresis. The expected number of cells in each reaction is indicated above each group of lanes. The marker (M) was $\phi$ X174 DNA digested with HaeIII. The PCR product is $261 \mathrm{bp}$ in length. 
unpublished data), we observed only four different sequences bounded by the primers used in this study. An additional 35 animals have now been examined by SSCP, six of which contain one of four variant sequences not originally observed. While both groups were decidedly nonrandom, there is no obvious reason why these new alleles should not have been present in the first group. More importantly, while an allele-specific, PCR-based assay would likely fail to detect all of the variant alleles described above, one of these, the apparent $\mathrm{O}$ to $\mathrm{A}$ revertant, could easily have been misidentified altogether. These considerations, combined with its ease of development, demonstrate the power and usefulness of two-color fluorescence SSCP for genotyping.

\section{ACKNOWLEDGMENTS}

We thank the Loma Linda Universi- ty and Medical Center, Immunology Center, and particularly Omar Fagoaga and Cathy Hisey, for providing washed lymphocytes from phenotyped baboons. This work was supported by LLUMC and the Department of Surgery.

\section{REFERENCES}

1.Bennett, E.P., R. Steffensen, H. Clausen, D.O. Weghuis and A.G. van Kessel. 1995. Genomic cloning of the human histo-blood group $\mathrm{ABO}$ locus. Biochem. Biophys. Res. Commun. 206:318-325.

2.Boutin, P., E.H. Hani, F. Vasseur, C. Roche, B. Bailleul, J. Hager and P. Froguel. 1997. Automated fluorescence-based screening for mutation by SSCP: use of universal M13 dye primers for labeling and detection. BioTechniques 23:358-362.

3.Clausen, H., E.P. Bennett and N. Grunnet. 1994. Molecular genetics of the ABO histoblood groups. Transfus. Clin. Biol. 2:78-89.

4.Crouse, C. and V. Vincek. 1995. Identification of $\mathrm{ABO}$ alleles on forensic-type specimens using rapid-ABO genotyping. BioTechniques 18:478-483.

5.Diamond, D.C., O. Fagoaga, S. NehlsenCannarella, L.L. Bailey and A.A. Szalay. 1997. Sequence comparison of baboon ABO histo-blood group alleles: lesions found in $\mathrm{O}$ alleles differ between human and baboon. Blood Cells Mol. Dis. 23:242-251.

6.Doxiadis, G.G., N. Otting, S.G., Antunes, N.G. de Groot, M. Harvey, I.I. Doxiadis, M. Jonker and R.E. Bontrop. 1998. Characterization of the ABO blood group genes in macaques: evidence for convergent evolution. Tissue Antigens 51:321-326.

7.El-Hashemite, N., D. Wells and J.D.A. Delhanty. 1997. Single cell detection of $\beta$-thalassaemia mutations using silver stained SSCP analysis: an application for preimplantation diagnosis. Mol. Hum. Reprod. 3:693-698.

8.Fukumori, Y., S. Ohnoki, H. Shibata, H. Yamaguchi and H. Nishimukai. 1995 Genotyping of ABO blood groups by PCR and RFLP analysis of 5 nucleotide positions. Int. J. Legal Med. 107:179-182.

9.Iwahana, H., M. Fujimura, Y. Takahashi, T. Iwabuchi and M. Itakura. 1996. Multiple fluorescence-based PCR-SSCP analysis using internal fluorescent labeling of PCR products. BioTechniques 21:510-519.

10.Kominato, Y., P.D. McNeill, M. Yamamoto, M. Russell, S. Hakomori and F. Yamamoto. 1992. Animal histo-blood group ABO genes. Biochem. Biophys. Res. Commun. 189:154164.

11.Kukita, Y., T. Tahira, S.S. Sommer and K. Hayashi. 1997. SSCP analysis of long DNA fragments in low $\mathrm{pH}$ gel. Hum. Mutat. 10:400-407.

12.Lee, J.C.-I. and J.-G. Chang. 1992. ABO genotyping by polymerase chain reaction. J. Forensic Sci. 37:1269-1275.

13.Liu, Q. and S.S. Sommer. 1998. The SSCP phenomenon: addition of HEPES buffer dramatically affects electrophoretic mobility.
BioTechniques 25:50-56.

14.Martinko, J.M., V. Vincek, D. Klein and J. Klein. 1993. Primate ABO glycotransferases: evidence for trans-species evolution. Immunogenetics 37:274-278.

15.Nehlsen-Cannarella, S.L. and M. Bohn. 1987. A direct approach to determine the $\mathrm{ABH}$ phenotype of baboons. Immunol. Invest. 16:57-62.

16.Ogasawara, K., M. Bannai, N. Saitou, R. Yabe, K. Nakata, M. Takenaka, K. Fujisawa, K. Fujisawa et al. 1996. Extensive polymorphism of $\mathrm{ABO}$ blood group gene: three major lineages of the alleles for the common ABO phenotypes. Hum. Genet. 97:777783

17.Ogasawara, K., R. Yabe, M. Uchikawa, N. Saitou, M. Bannai, K. Nakata, M. Takenaka, K. Fujisawa et al. 1996. Molecular genetic analysis of variant phenotypes of the $\mathrm{ABO}$ blood group system. Blood 88:2732-2737.

18.O'Keefe, D.S. and A. Dobrovic. 1993. A rapid and reliable PCR method for genotyping the ABO blood group. Hum. Mutat. 2:67-70.

19.Olsson, M.L. and M.A. Chester. 1995. A rapid and simple $\mathrm{ABO}$ genotype screening method using a novel $\mathrm{B} / \mathrm{O}^{2}$ versus $\mathrm{A} / \mathrm{O}^{2}$ discriminating nucleotide substitution at the ABO locus. Vox Sang. 69:242-247.

20.Ray, P.F. and A.H. Handyside. 1997. PCR from single cells for preimplantation diagnosis. p. 245-258. In R. Elles (Ed.), Methods in Molecular Medicine: Molecular Diagnosis of Genetic Diseases. Humana Press, Totowa, NJ.

21.Szalay, A.A., K. Grohmann and R.L. Sinsheimer. 1977. Separation of the complementary strands of DNA fragments on polyacrylamide gels. Nucleic Acids Res. 4:1569-1578.

22.Ugozzoli, L. and R.B. Wallace. 1992. Application of an allele-specific polymerase chain reaction to the determination of $\mathrm{ABO}$ blood group genotypes. Genomics 12:670-674.

23. Yamamoto, F. 1995. Molecular genetics of the ABO histo-blood group system. Vox Sang. 69:1-7.

24.Yamamoto, F., H. Clausen, T. White, J. Marken and S. Hakomori. 1990. Molecular genetic basis of the histo-blood group $\mathrm{ABO}$ system. Nature 345:229-233.

25.Yamamoto, F., P.D. McNeill and S. Hakomori. 1995. Genomic organization of the human histo-blood group ABO genes. Glycobiology 5:51-58.

Received 30 April 1999; accepted 7 July 1999.

Address correspondence to:

Dr. David Diamond

Center for Molecular Biology

and Gene Therapy

Dr. Aladar Szalay

Department of Biochemistry

Loma Linda University

and Medical Center

Loma Linda, CA 92354, USA

Internet: rddiamond@earthlink.net 